\title{
Non-destructive assay of nuclear waste containers using muon scattering tomography in the Horizon2020 CHANCE project
}

\author{
Anna Kopp $^{1 \star}$, Ahmad Alrheli ${ }^{2}$, Daniel Kikoła ${ }^{3}$, Mohammed Mhaidra ${ }^{13}$, Patrick Stowell ${ }^{2}$, \\ Holger Tietze-Jaensch ${ }^{4}$, Lee Thompson ${ }^{2}$, Jaap Velthuis ${ }^{1}$, Michael Weekes ${ }^{2}$ \\ ${ }^{1}$ School of Physics, University of Bristol, UK \\ 2 Department of Physics and Astronomy, University of Sheffield, UK \\ ${ }^{3}$ Warsaw University of Technology, Poland \\ ${ }^{4}$ Forschungszentrum Jülich, Germany \\ * anna.kopp@bristol.ac.uk
}

\begin{abstract}
Methods for the non-destructive assay of nuclear waste drums are of great importance to the nuclear waste management community, especially where loss in continuity of knowledge about the content of drums happened or chemical processes altering the contents of the drums may occur. Muon scattering tomography has been shown to be a promising technique for the non-destructive assay of nuclear waste drums in a safe way. By measuring tracks of muons entering and leaving the probed sample and extracting scattering angles from the tracks, it is possible to draw conclusions about the contents of the sample and its spatial arrangement. Within the CHANCE project, a newly built large-scale mobile detector system for scanning and imaging the contents of nuclear waste drums using atmospheric muons is currently undergoing commissioning.
\end{abstract}

\section{INTRODUCTION}

Non-destructive methods to assay nuclear waste drums are of great interest to the nuclear waste management community. It has been observed that chemical processes like oxidation of metals may occur within drums, possibly leading to the formation of gas bubbles or cracks. Furthermore, knowledge about the contents of legacy waste drums is not always preserved. Muon scattering tomography (MST) is a promising technique to address these problems. It allows to scan and image nuclear waste drums in a safe, non-destructive way using natural background radiation. Compared to other methods like Xray or gamma-ray scanning it does not introduce additional artificial radiation or any additional hazards to personnel or equipment. Fitting the tracks of muons entering and leaving the probed sample allows to reconstruct approximated scattering vertices and to e.g. differentiate between various materials.

\section{Muon ScAtTERING TOMOgRAPHY}

Muon scattering tomography uses secondary cosmic radiation to probe volumes from a safe distance. Compared to other scanning methods using e.g. X-rays or gamma rays it does not rely on a radiation source but uses atmospheric muons, particles resulting from primary cosmic radiation. These are ubiquitous and abundant at a rate of about $10000 /\left(\mathrm{m}^{2}\right.$ minute $)$ at sea level, spread over a wide range of momenta and incidence angles. Muons are highly penetrating particles; it is almost impossible to stop them and they are thus ideally suited for scanning nuclear waste drums, where the nuclear waste is often embedded in concrete.

As charged particles, they undergo multiple Coulomb scattering processes when traversing matter. The projected scattering angle distribution depends on the atomic number $Z$ of the traversed material and can be approximated as a Gaussian distribution [1] with mean zero and a standard deviation $\sigma_{\mu}$ of

$$
\sigma_{\mu} \approx \frac{13.6 \mathrm{MeV}}{p c \beta} \sqrt{X / X_{0}}\left(1+0.038 \ln \left(X / X_{0}\right)\right),
$$

where $p$ is the muon's momentum, $\beta c$ its velocity, $X$ the thickness of the scattering material and $X_{0}$ the materialspecific radiation [2] length given by

$$
X_{0} \approx \frac{A \cdot 716.4 \mathrm{~g} / \mathrm{cm}^{2}}{Z(Z+1) \ln (287 / \sqrt{Z})} .
$$

Here, $A$ is the atomic weight given in $\mathrm{g} / \mathrm{mol}$. As can be seen from equations 1 and 2, the width of the projected scattering angle distribution varies approimately with $Z$, making the technique particularly sensitive to materials with high atomic numbers.

Thus scattering angles from muons scattering off materials with large atomic numbers $Z$ are more likely to be large than those from scatters in low- $Z$ materials.

By placing multiple detector planes above and below the probed sample and measuring where the muons hit these, the trajectories of the incoming and outgoing muons are reconstructed and fitted. A scattering vertex is then reconstructed where these two trajectories meet. The assumption here that for each muon all scattering processes happen in the same location, the vertex, is not strictly correct but has been shown to be a good approximation. Scattering angles for all muons are derived from their fitted tracks.

Then, the volume under investigation is divided into voxels as described in ref. [4]. In each voxel with at least $N$ 


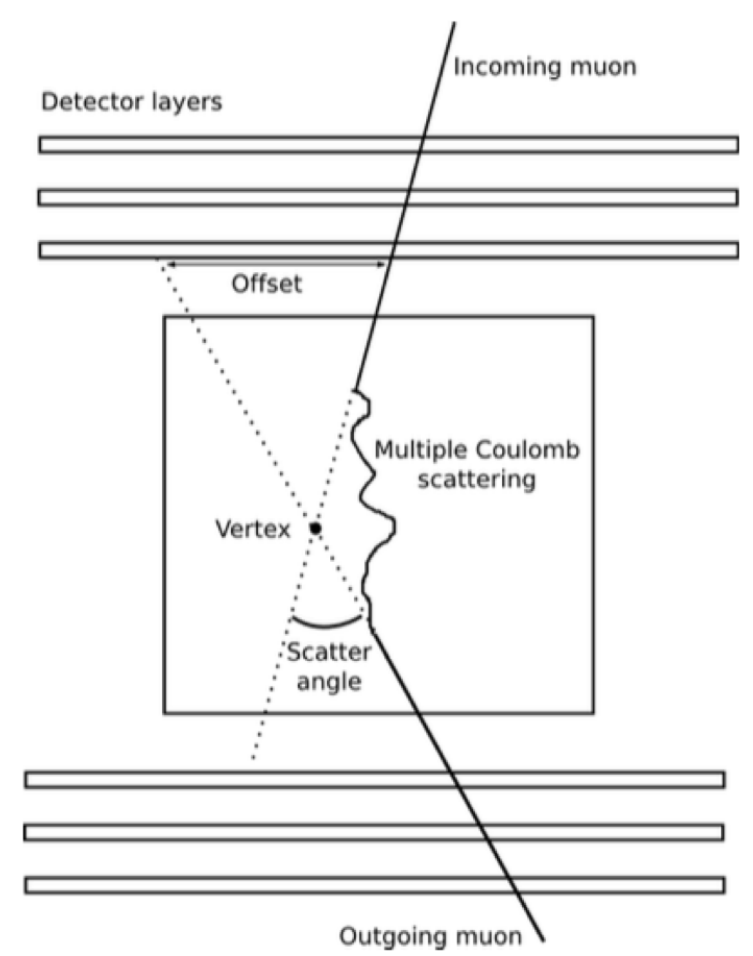

Fig. 1. Illustration of the muon trajectory and the reconstructed scattering vertex [3].

reconstructed vertices, a metric discriminator $m_{i j}$ is calculated for each pair of tracks $i$ and $j$ of the $N$ most scattered tracks,

$$
m_{i j}=\frac{\left\|\vec{v}_{i}-\vec{v}_{j}\right\|}{\left(\theta_{i} p_{i}\right)\left(\theta_{j} p_{j}\right)} .
$$

Here, $\vec{v}_{i}$ is the location of the vertex pertaining to muon track $i$ with momentum $p_{i}$ and $\theta_{i}$ is the associated scattering angle. The median of this weighted metric distribution per voxel is an indicator of the predominant material in that voxel: large values are more likely to result from materials with high $Z$ than from materials with low $Z$.

In the past, this has been exploited for various discrimination and identification studies in simulation: to differentiate between different high- $Z$ materials [3], [5] or to locate gas bubbles in bitumen-filled drums [6].

\section{THE CHANCE PROJECT}

The CHANCE project ("Characterization of conditioned nuclear waste for its safe disposal in Europe", http://www. chance-h2020.eu), is funded by the EU's Horizon2020 research programme. It started on June 1st 2017, runs over 4 years and has 11 partners in 7 countries. Collaborators include universities and research institutes as well as government agencies and industrial partners. Within the project, three different techniques to assay nuclear waste drums in nondestructive ways are investigated: calorimetry, muon scattering tomography and cavity ring-down spectroscopy.

\section{Muon Scattering TOMOgRaphy Within the CHANCE PROJECT}

Three universities mainly contribute to the MST program within CHANCE: University of Sheffield, University of Bristol, both in the UK and Warsaw University of Technology, Poland. A large, mobile muon scattering tomography detector shown in figure 2 has been recently built in a non-laboratory environment outside of Bristol, UK. It is placed in a building, so it is rain-protected but the building is neither temperaturenor humidity-controlled. Thus the environmental conditions are the same as they are likely to be in an actual field deployment. The active area measures approximately $1.8 \times 1.8 \mathrm{~m}^{2}$. As the detector system is modular, it is easy to dis- and reassemble and even to change the configuration to adapt it to the requirements in a potential deployment.

The detector system combines two different kinds of gas detectors, namely drift chambers and resistive plate chambers (RPCs). A coincidence between two layers of plastic scintillators is used as trigger to start the read-out process.

The RPCs were designed and built at Univeristy of Bristol. A smaller prototype of these chambers has been operated on a mixture of freon (R134-a) and isobutane in the past. Due to recent legislation restricting the usage of freon, the chambers are currently run on $\mathrm{CO}_{2}$. Other, environmentally friendly gas mixtures will be tested in the future. The drift chambers are operated on a mix of methane, argon and $\mathrm{CO}_{2}$. They were built at University of Sheffield.

During the construction of the detector system the data analysis effort had been focussed on simulation studies. The detector layout was optimized and a figure of merit was developed for comparing the smallest observable separations between features or the smallest observable features in concrete-filled waste drums using different reconstruction algorithms [7]. The latter is especially relevant considering the heterogeneity of real nuclear waste drums. Commissioning of the full detector system has started in early June and is ongoing, with first test data having been taken. Full data taking is expected to start soon while further simulation studies are continuing in parallel.

\section{The Detector System}

Both RPCs and drift chambers provide information in $3 \mathrm{D}$ space about where a muon hit the detector. As the muon traverses a detector chamber, the gas inside it gets ionized. With a high voltage applied between the top and bottom side for the RPCs or the anode wire and cathode for the drift chambers, respectively, this creates a signal, which is read out and digitized. The spatial resolution of the RPCs is in the submillimeter range [8], while that of the drift chambers is in the order of $\mathrm{mm}$. The time resolution for RPCs with a $2 \mathrm{~mm}$ gap as the ones used here is on the order of nanoseconds.

In both the detector stack above and below the sample space, two space points from the RPCs and three from the drift chambers are measured and read out. Hence it is possible to fit tracks and extract scattering angles with high precision. The detectors are fast, with data acquisition per event taking 


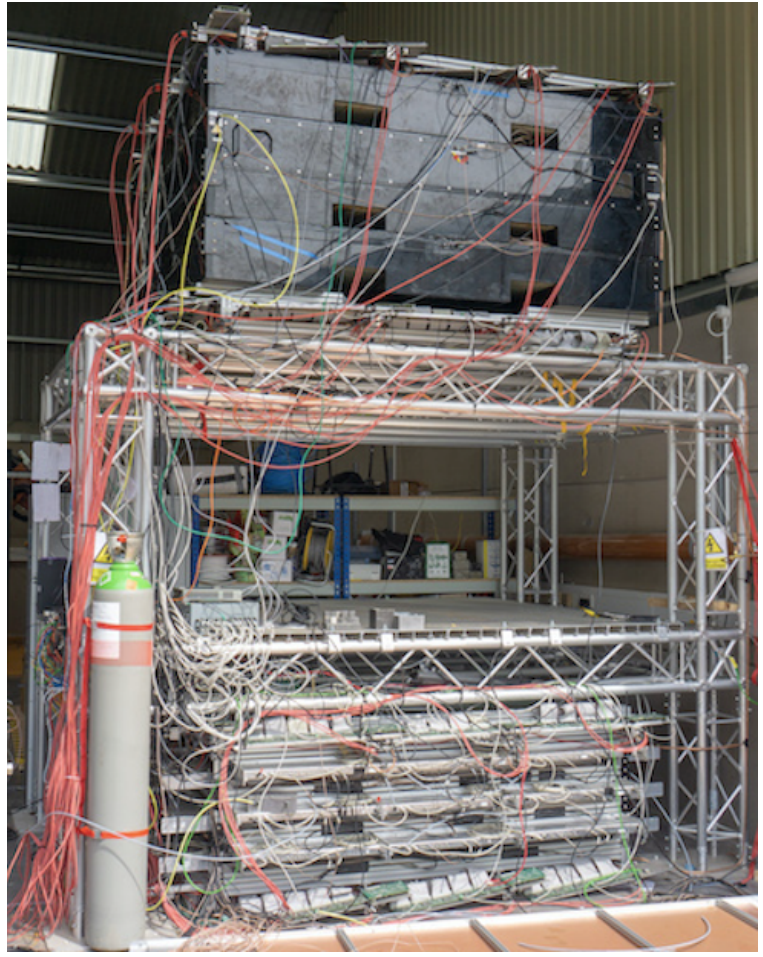

Fig. 2. The muon scattering tomography detector system of the CHANCE project. Shown are the detector systems above and below the empty sample space consisting of three layers of drift chambers and two RPC layers. The top stack additionally contains two layers of plastic scintillators used as triggers. The detectors cover an area of approximately $1.8 \times 1.8 \mathrm{~m}^{2}$.

on the order of a few $10 \mu$ s and have a low cost per unit area, making them ideal for large-scale detectors. For both the RPCs and drift chambers, three individual detectors with widths of 58 and $60 \mathrm{~cm}$, respectively, are placed next to each other to cover the whole active area. The support structure holding the individual chambers can be seen as empty space in the data as shown for one layer of RPCs in figure 3. Since the footprint of the active area is larger than the drums that will be scanned, it is nonetheless possible to scan whole drums and avoid dead areas by moving the drum to different locations within the sample space.

\section{CONCLusions And OutlooK}

Muon scattering tomography is a non-destructive technology well suited to investigate the contents of nuclear waste drums in a safe way, without introducing any additional radiation to the samples or personnel. A large, mobile MST detector has been built in a non-laboratory environment close to Bristol, UK, within the CHANCE project and first test data have been taken. Once the detector system is fully commissioned, full data taking will commence.

As the scattering behavior of atmospheric muons in many materials is well known from simulation studies, data with blocks of high- $Z$ materials like lead or tungsten will be taken in a first step. Subsequently, 'blind tests' with drums mimicking nuclear waste drums will be carried out. Our collaboration partners at SCK·CEN, Belgium, filled drums

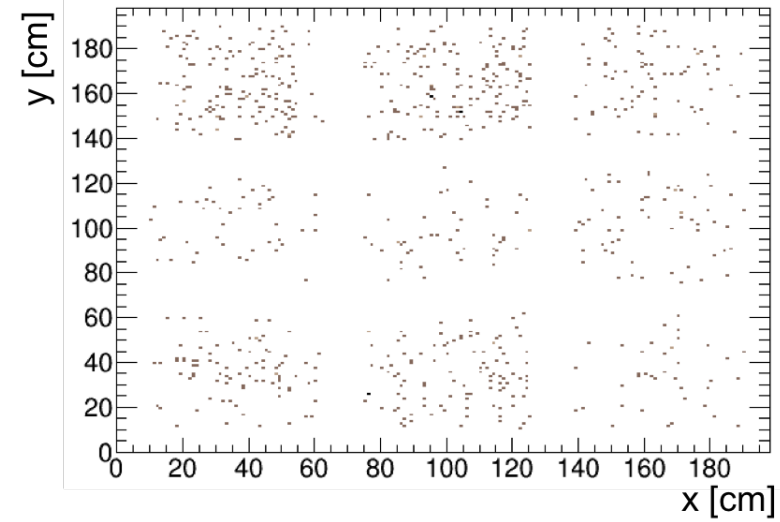

Fig. 3. Hits in one layer of RPCs. The pattern is due to the support structure holding the individual detector chambers. Each entry corresponds to one hit, showing the $x$ and $y$ coordinates.

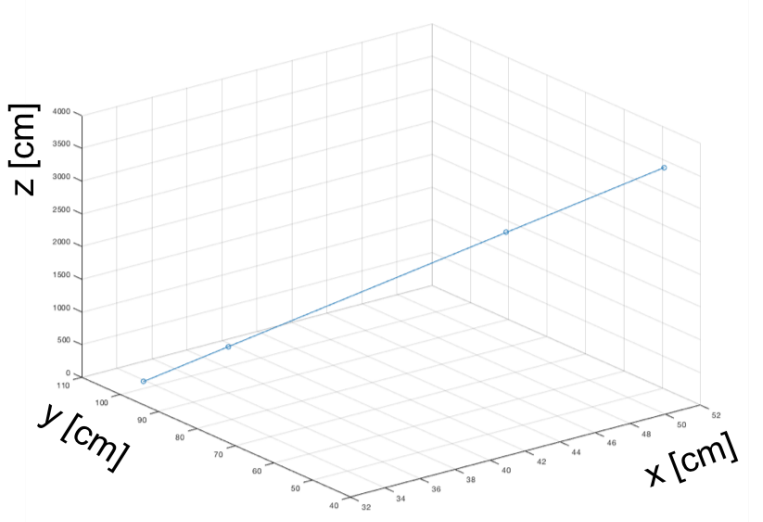

Fig. 4. Muon track fitted through all four layers of RPCs. No sample is placed between the upper and lower detectors so no scattering is expected or seen.

with non-radioactive materials otherwise similar to what could be found in nuclear waste drums but did not reveal the contents. Scanning the drums and applying the reconstruction algorithm described above will then show their contents. The access and application to real waste drums is currently being investigated.

\section{ACKNOWLEDGMENT}

This project has received funding from the European Union's Horizon2020 research and innovation programme under grant agreement number 755371 .

\section{REFERENCES}

[1] M. Tanabashi et al., "Review of particle physics," Phys. Rev. $D$, vol. 98, p. 030001, Aug 2018. [Online]. Available: https: //link.aps.org/doi/10.1103/PhysRevD.98.030001

[2] T. A. Lasinsky et al., "Review of particle properties particle data group," Rev. Mod. Phys., vol. 45, pp. S1-S175, Apr 1973. [Online]. Available: https://link.aps.org/doi/10.1103/RevModPhys.45.S1 
[3] L. Frazão, J. Velthuis, C. Thomay, and C. Steer, "Discrimination of high-Z materials in concrete-filled containers using muon scattering tomography," Journal of Instrumentation, vol. 11, no. 07, pp. P07 020-P07 020, Jul 2016. [Online]. Available: https://doi.org/10.1088/ $1748-0221 / 11 / 07 / \mathrm{p} 07020$

[4] C. Thomay, J. J. Velthuis, P. Baesso, D. Cussans, P. A. W. Morris, C. Steer, J. Burns, S. Quillin, and M. Stapleton, "A binned clustering algorithm to detect high-z material using cosmic muons," Journal of Instrumentation, vol. 8, no. 10, pp. P10013-P10 013, Oct 2013. [Online]. Available: https://doi.org/10.1088/1748-0221/8/10/P10013

[5] C. Thomay, J. Velthuis, T. Poffley, P. Baesso, D. Cussans, and L. Frazão, "Passive 3d imaging of nuclear waste containers with muon scattering tomography," Journal of Instrumentation, vol. 11, no. 03, pp. P03 008-P03 008, Mar 2016. [Online]. Available: https: //doi.org/10.1088/1748-0221/11/03/p03008

[6] M. Dobrowolska, J. Velthuis, L. Frazão, and D. Kikoła, "A novel technique for finding gas bubbles in the nuclear waste containers using muon scattering tomography," Journal of Instrumentation, vol. 13, no. 05, pp. P05 015-P05 015, May 2018. [Online]. Available: https://doi.org/10.1088/1748-0221/13/05/p05015

[7] J. Stowell, J. Velthuis, H. Tietze-Jaensch, A. Kopp, A. Alrheli, M. Mhaidra, L. Thompson, and D. Kikoła, "Figures of merit for the application of muon tomography to the characterization of nuclear waste drums," Waste Management Symposia 2019, no. 19253, March 2019.

[8] P. Baesso, D. Cussans, C. Thomay, and J. Velthuis, "Toward a RPC-based muon tomography system for cargo containers." Journal of Instrumentation, vol. 9, no. 10, pp. C10041-C10 041, Oct 2014. [Online]. Available: https://doi.org/10.1088/1748-0221/9/10/c10041 